\title{
Symptom Burden and Quality of Life After Successful Ablation in Patients With Low Burden of Symptomatic Premature Ventricular Complexes
}

muzakkir amir ( $\nabla$ muzakkir@unhas.ac.id )

Hasanuddin University

\section{Peter Kabo}

Hasanuddin University

Pendrik Tandean

Hasanuddin University

Ilham Patellongi

Hasanuddin University

Muchtar Siregar

Hasanuddin University

Sitti Zam

Hasanuddin University

\section{Research Article}

Keywords: Symptom Burden, Ventricular, Low Burden

Posted Date: January 4th, 2022

DOI: https://doi.org/10.21203/rs.3.rs-956291/v2

License: (1) This work is licensed under a Creative Commons Attribution 4.0 International License.

Read Full License 


\section{Abstract}

It is essential to enhance life quality in patients with premature ventricular complexes (PVCs) with the use of radiofrequency ablation (RFA). The aim of the study was to assess symptom burden and life quality in patients with a low PVC burden following RFA. 31 individuals with a low PVC burden in whom RFA was indicated were included in the study. At baseline and after a year following RFA, the Arrhythmia-Specific questionnaire in Tachycardia and Arrhythmia (ASTA) scale was used for appraisal. A 24-hour Holter electrocardiogram was used to detect recurrent PVCs 12 months after the RFA intervention. ASTA scores related to symptom burden, including the near-syncope score, health-related quality of life (HRQOL) scales pertaining to physical and mental health, and consequently the total HRQOL score, were all diminished a year after RFA ( $\mathrm{p}<0.001)$. The ASTA score for syncope symptoms was also reduced $(p<0.05)$. A fall in mean PVC burden was seen from $8.0 \%$ to $0.8 \%(p<0.001)$. The long-term clinical endpoint in individuals with a low PVC burden following RFA is reported. Symptom load, life quality and ultimate PVC burden were all enhanced. Additional studies incorporating longer follow-up and monitoring periods, respectively, would be beneficial.

\section{Introduction}

In patients with premature ventricular complexes (PVCs), life quality can be enhanced by radiofrequency ablation (RFA) ${ }^{1}$. Objectives of cardiac rehabilitation include diminishing symptomatology and attaining superior levels of physical activity and overall health ${ }^{2}$.

In routine clinical work, PVCs are the most frequent cardiac dysrhythmia encountered, with a prevalence of $1.1 \%$ being documented in a telemedicine study performed in Makassar City ${ }^{3}$. A further study noted PVCs in $1 \%$ and $40-75 \%$ of individuals on 12 -lead and Holter electrocardiography, respectively ${ }^{4}$. PVCs are more common in patients of more advanced years; frequencies of a minimum of one PVC detected on 24 -hour cardiac recordings were $16.7 \%$ in well individuals below the age of 30 years, and up to $69 \%$ in those above the age of 75 years ${ }^{5}$.

Within the overall population, the impact of PVCs on the establishment of congestive cardiac impairment or fatality is unclear. Nevertheless, a number of publications have implied that the likelihood of sudden cardiac demise, cardiovascular morbidity and systolic left ventricular impairment may be exacerbated by numerous PVCs ${ }^{6}$. In addition, patients may present with palpitations, fatigue, chest discomfort, dizziness, loss of conscious and symptoms of cardiac impairment. Although these issues are common in patients with a high burden of PVCs, low PVC frequency, i.e. < 5000/24 hours, can also generate significant symptomatology and merit treatment ${ }^{7}$.

Earlier clinical studies have demonstrated that an impairment in left ventricular ejection fraction (LVEF) may be associated with a higher than mean burden of PVCs. Interestingly, the frequency of PVCS did not always predict left ventricular function, with patients with a high PVC burden displaying normal cardiac performance whereas cardiomyopathy related to PVCs was at times seen in subjects with a relatively 
small PVC burden ${ }^{8-10}$. Currently, no specific estimate of PVC burden has been determined to be a reliable predictor of cardiac dysfunction ${ }^{711-13}$ although an arbitrary cut-off parameter of $10 \%$ PVCs with respect to the overall heart rate, as opposed to the absolute PVC frequency, has been employed in a number of studies ${ }^{1114}$.

RFA is an efficacious therapeutic intervention for PVCs. Contemporary pharmaceutical agents for dysrhythmias are not always effective and may have an adverse side effect profile ${ }^{15}$. Clinical recommendations published by the European Society of Cardiology (2015) and the American Heart Association/American College of Cardiology (2017) relating to the care of individuals with ventricular dysrhythmia and sudden cardiac death prevention advise the use of RFA in patients who present with PVCs that give rise to symptoms, reduced LVEF and in whom anti-arrhythmic agents fail ${ }^{1617}$.

A number of studies have provided evidence regarding the non-subjective positive endpoints following the use of RFA in individuals with a high PVC burden, although PVCs were noted to recur after a post-

procedural surveillance period of at least six months ${ }^{811} 1218$. One retrospective study followed up 44 individuals who had received RFA for a symptomatic high PVC burden for over a year; monitoring determined a positive outcome rate following RFA of $75 \%{ }^{19}$. PVCs, arising from either a different or identical pre-ablation focus, recurred in $18.2 \%$ and $6.8 \%$ patients, respectively.

RFA does not influence mortality statistics, and therefore its clinical endpoints should be judged in relation to subjective changes in patient symptomatology. Few studies have evaluated the application of RFA in individuals who have few PVCS, particularly with respect to their symptoms and life quality. A low PVC burden may provoke symptomatology; some individuals may exhibit clinical evidence of reduced LVEF. The current research has appraised the utility of RFA with respect to the symptomatic load and life quality of individuals with symptoms pertaining to a low PVC burden. The data presented can be utilized as a foundation for establishing the utility of RFA in the clinical care of this patient cohort.

\section{Methods}

\section{Study design}

An observational, single-centre, cohort study was performed in order to assess the outcome of individuals with a low burden of PVCs treated at either the Medical Faculty of Hasanuddin University or the Dr. Wahidin Sudirohusodo Hospital, Makassar, Indonesia. The study was conducted between January and December, 2019. The study was approved by Commission on Health Research Ethics Medical Faculty of Hasanuddin University RSPTN with protocol number UH20020106. All participant will be informed of the research aims and objectives, and that confidentiality will be maintained throughout the study. Information and consent form provided at baseline and participant will be free to withdraw from the research at any time. All methods were performed in accordance with the Declaration of Helsinki. 


\section{Patient characteristics}

All 31 study participants had a low PVC burden, as evaluated by 24-hour Holter electrocardiography and defined as $\leq 10 \%$ PVCs per 24-hour period. Indications for RFA included continuing symptoms despite use of anti-arrhythmic agents, failure to withstand medication and patient choice. Study exclusion criteria encompassed those who had undergone former RFA or who had additional coexistent rhythm disturbances. Patient demographics were documented, i.e., gender, age, weight, height, body mass index (BMI) and additional comorbidities.

\section{Echocardiography}

2-dimensional echocardiography was carried out before and after RFA intervention. LVEF and left ventricular end-diastolic dimension (LVEDd) were computed according to the Simpson method from an M-mode image obtained in the parasternal long axis plane.

\section{Radiofrequency ablation}

Following written informed consent, all patients underwent electro anatomical mapping and radiofrequency catheter ablation of the PVC foci per standard laboratory protocol and the judgment of the operator. Mapping and ablation were performed with 7Fr quadripolar electrodes and a deflectablecurve, temperature-guided ablation catheter. Activation mapping was used with 3-dimensional mapping system. Attempts were made to record an earliest endocardial activation at least $30 \mathrm{~ms}$ before the onset of the QRS complex from the ectopic site. The aim was to achieve a 11/12 or 12/12 identical pace mapping. Once VPCs were localized, radiofrequency power of 20 to $40 \mathrm{~W}$ to maintain the temperature at the catheter tip of $50^{\circ} \mathrm{C}$ was delivered. After ablation, all patients were kept under observation for 30 min. Successful ablation was defined as complete elimination of spontaneous or inducible PVC ${ }^{1}$.

\section{Arrhythmia-Specific questionnaire in Tachycardia and Arrhythmia scale}

The Arrhythmia-Specific questionnaire in Tachycardia and Arrhythmia scale (ASTA) scale was utilized in order to evaluate subjects' symptom load and impact on life quality prior to and 12 months after RFA, the latter in conjunction with a further 24-hour Holter electrocardiogram in order to identify recurrent PVCs. The questionnaire was split into three sections, which assessed: (i) the most recent arrhythmic event and ongoing drug therapy; (ii) the symptom load according to a 9-item 4-point response scale (the ASTA symptom scale); and (iii) life quality using a 6- and a 7-item subscale, i.e. 13 items in total, comprising similar 4-point response rankings, i.e. the ASTA Health-Related Quality of Life (HRQOL) scale. The second section also encompassed enquiries regarding the prevalence of events relating to PVCs, the mean length of symptoms and the most enduring event, together with any history of associated loss of 
consciousness, dizziness or palpitations. The ASTA HRQOL evaluation generated scores ranging between 0 and 100; the latter indicated a high symptom load and greater impact of the PVC burden on life quality 2021 .

\section{Statistical analysis}

The Statistical Package for the Social Sciences software was used for data analysis. Mean \pm standard deviation, together with minimal and maximal scores are presented for the continuous variables; frequencies are provided for the categorical data. Life quality pre- and post-RFA was compared using a paired simple t-test. Significance was defined as a $p$ value $<0.05$.

\section{Results}

The study sample included 19 females (61.4\%) and 12 males (38.7\%). Mean age was 43.0 years; age range was $17-74$ years. Mean weight was $61.7 \mathrm{~kg}$, height, $160.0 \mathrm{~cm}$, and BMl, $24.3 \mathrm{~kg} / \mathrm{cm}^{2}$. Comorbid factors were absent in $51.6 \%$; hypertension, coronary heart disease and diabetes mellitus were present in $38.7 \%, 6.5 \%$ and $3.2 \%$, respectively. Anti-arrhythmic medication was prescribed prior to RFA; 26 patients (83.9\%) were on beta blockers, 4 (12.9\%) were on amiodarone, and $1(3.2 \%)$ was receiving a calcium channel blocker.

A 24-hour Holter monitor was conducted prior to the RFA intervention. The majority of PVCs, $45.2 \%$, originated from the anteroseptal right ventricular outflow tract (RVOT). Additional common foci included the posteroseptal RVOT in $38.7 \%$ and anteroseptal left ventricular outflow tract (LVOT) in $6.5 \% .3 .2 \%$ subjects exhibited PVCs derived from the anterolateral RVOT, posterior RVOT and HIS. Patient demographics are illustrated in Table 1.

\section{Table 1. Patient characteristics}




\begin{tabular}{|c|c|c|c|c|}
\hline Variables & $\operatorname{Min} / \mathbf{M a x}$ & Mean \pm SD & $\begin{array}{l}\text { Number of } \\
\text { respondents } \\
(n=31)\end{array}$ & $\%$ \\
\hline \multicolumn{5}{|l|}{ Sex } \\
\hline Male & & & 12 & 38.7 \\
\hline Female & & & 19 & 61.3 \\
\hline Age (years) & $17 / 74$ & $43.0 \pm 14.4$ & & \\
\hline Weight (kg) & $43 / 84$ & $61.7 \pm 9.8$ & & \\
\hline Height $(\mathrm{cm})$ & $149 / 173$ & $160.0 \pm 6.7$ & & \\
\hline $\mathrm{BMI}\left(\mathrm{kg} / \mathrm{cm}^{2}\right)$ & $17.7 / 30.8$ & $24.3 \pm 3.7$ & & \\
\hline \multicolumn{5}{|l|}{ Comorbid factors } \\
\hline None & & & 16 & 51.6 \\
\hline Coronary heart disease & & & 2 & 6.5 \\
\hline Hypertension & & & 12 & 38.7 \\
\hline Diabetes mellitus & & & 1 & 3.2 \\
\hline \multicolumn{5}{|l|}{ Anti-arrhythmic drugs } \\
\hline Beta blocker & & & 26 & 83.9 \\
\hline Calcium channel blocker & & & 1 & 3.2 \\
\hline Amiodarone & & & 4 & 12.9 \\
\hline \multicolumn{5}{|l|}{ PVC locations } \\
\hline RVOT anteroseptal & & & 14 & 45.2 \\
\hline RVOT posteroseptal & & & 12 & 38.7 \\
\hline RVOT posterior & & & 1 & 3.2 \\
\hline RVOT anterolateral & & & 1 & 3.2 \\
\hline LVOT anteroseptal & & & 2 & 6.5 \\
\hline HIS & & & 1 & 3.2 \\
\hline
\end{tabular}

Echocardiographic findings from baseline and a year after RFA are displayed in Table 2. Mean baseline and 12-month follow-up LVEFs were 62.9\% (29.0-76.0\%) and 64.2\% (35.5-73\%), respectively. Pre- and 12month post-ablation LVEDd were $43.8 \mathrm{~mm}(36.0-67.0 \mathrm{~mm})$ and $45.0 \mathrm{~mm}(40.0-67.0 \mathrm{~mm})$. No differences were detected following the intervention. Prior to the procedure, the mean PVC burden was $8.0 \%$. In contrast, 12 months following RFA, the mean PVC burden detected was markedly reduced, i.e. $0.8 \%$ (range: $0-5.6 \%)(p<0.001)$.

Table 2. Comparison of echocardiographic parameters and PVC burden before and after ablation

\begin{tabular}{cccccc}
\hline Variable & Min-Max & Mean \pm S.D & Min-Max & Mean \pm S.D & p value* \\
\hline Ejection fraction (\%) & $29.0-76.0$ & $62.9 \pm 7.7$ & $35.5-73.0$ & $64.2 \pm 6.91$ & 0.505 \\
\hline LVEDd (mm) & $36.0-67.0$ & $43.8 \pm 5.3$ & $40.0-67.0$ & $45.0 \pm 5.22$ & 0.384 \\
\hline PVC burden (\%) & $4.3-10.0$ & $8.0 \pm 1.5$ & $0.0-5.6$ & $0.80 \pm 1.49$ & $<0.001$ \\
\hline
\end{tabular}

\section{*Paired t-test for comparison before and after ablation}

\section{Changes in symptom burden and quality of life 12 months after radiofrequency ablation}

The symptom load evaluated with the use of the ASTA score demonstrated a reduction from 39.1 to 2.1 (range: 0-21.0) following RFA (Table 3). Baseline ASTA near-syncope score was 7.5; this dropped to 0 following the intervention, with no participants reporting symptoms of near-syncope or syncope during the surveillance period. All the symptom burdens measured on the ASTA score decreased $(p<0.05)$. 
Life quality was also evaluated at baseline and at one year following RFA, yielding HRQOL physical subscale values of 30.7 and 0.3 (range: 0-9.1), respectively. The mental HRQOL additionally diminished following RFA, with baseline and post-intervention values of 32.7 and 2.7 (range: 0-21.1), respectively. Thus, post-RFA, the total HRQOL scale score decreased from 32.5 to 1.5 , indicating a notable improvement in life quality $(p<0.001)$.

Table 3. Symptom burden and quality of life before and 12 months after ablation

\begin{tabular}{cccccc}
\hline Variable & Min-Max & Mean \pm S.D & Min-Max & Mean \pm S.D & p value* \\
\hline $\begin{array}{c}\text { ASTA symptom } \\
\text { burden }\end{array}$ & $17.8-75.0$ & $39.1 \pm 14.98$ & $0.0-21.0$ & $2.1 \pm 4.34$ & $<0.001$ \\
\hline $\begin{array}{c}\text { ASTA symptom } \\
\text { near-syncope }\end{array}$ & $0.0-75.0$ & $7.5 \pm 22.54$ & $0.0-0.0$ & $0.00 \pm 0.00$ & $<0.001$ \\
\hline $\begin{array}{c}\text { ASTA symptom } \\
\text { syncope }\end{array}$ & $0.0-85.7$ & $2.8 \pm 15.39$ & $0.0-0.0$ & $0.00 \pm 0.00$ & $<0.005$ \\
\hline $\begin{array}{c}\text { Physical subscale } \\
\text { HRQOL }\end{array}$ & $4.5-86.3$ & $30.70 \pm 17.72$ & $0.0-9.1$ & $0.30 \pm 1.66$ & $<0.001$ \\
\hline $\begin{array}{c}\text { Mental subscale } \\
\text { HRQOL }\end{array}$ & $15.7-89.4$ & $32.7 \pm 21.52$ & $0.0-21.1$ & $2.7 \pm 5.46$ & $<0.001$ \\
\hline $\begin{array}{c}\text { Total scale } \\
\text { HRQOL }\end{array}$ & $10.0-87.5$ & $32.5 \pm 19.37$ & $0.0-12.5$ & $1.50 \pm 3.12$ & $<0.001$ \\
\hline
\end{tabular}

\section{*Paired t-test for comparison before and after ablation}

\section{Recurrence of PVCs 12 months after ablation}

If the burden of PVCs remained over $80 \%$ of the baseline value on the 24-hour Holter electrocardiogram after 12 months or PVCs noted on the monitor were symptomatic, the RFA was deemed to be a failure and PVCs to have recurred. Ten of the 30 participants who underwent RFA exhibited PVCs on the followup 24-hour Holter recording, of whom 5 were described as having a good ablation outcome with an $80 \%$ reduction in PVCs, and 5 were considered to have a poor procedural endpoint, with a PVC burden fall of less than $80 \%$. Eight subjects fulfilled the parameters for PVC recurrence, whereas the remaining two did not as their PVCs had been ameliorated by over $80 \%$. Despite the evident PVCs, these individuals reported no symptoms. Thus, the proportion of subjects who presented with recurrent PVCs was documented to be $26.6 \%(8 / 30)$ (Table 4).

Table 4. Distribution of subjects showing PVCs 12 months after ablation and symptoms category

\begin{tabular}{lccc}
\hline Ablation results category & $\mathbf{n}=\mathbf{1 0}$ & Symptomatic & Asymptomatic \\
\hline Good $(\geq 80 \%)$ & $5(50 \%)$ & 3 & 2 \\
\hline Poor $(<80 \%)$ & $5(50 \%)$ & 3 & 2 \\
\hline
\end{tabular}

\section{Discussion}

Characteristics of symptomatic low burden PVC patients undergoing ablation procedures 
In this patient sample, the proportion of females experiencing symptoms from a low PVC burden was higher than males. Gender differences in the prevalence of PVCs have been reported in previous studies, e.g. the ARIC study and a study published by Kerola et al. ${ }^{22}$; both concluded that being male increased the likelihood of PVCs. The results from the current study concur with several studies pertaining to the incidence of PVCs 232425 . Of 30 patients with numerous PVCs who were treated with RFA, females reported a higher number of related symptoms. It is possible that the latter show a heightened awareness of milder disturbances associated with PVCs and are therefore more likely to present than males, although to date, there are no data to support this theory.

PVCs can be detected in individuals at any life stage, but research has implied that they become more common with advancing years. In this study, the mean age of the population with low burden PVCs was over 40 years. A similar mean age was noted by Darrieux et al. ${ }^{26}$ in 30 patients who were treated with RFA for symptomatic multiple PVCs. The observation of age being a predictor of symptomatic PVC prevalence was also reported by a number of other researchers ${ }^{242527}$, and could be associated with the overall heightened cardiovascular risk profile in the elderly, together with the onset of additional generalized symptomatology that leads individuals to seek clinical review.

The PVC site is strongly associated with symptom prevalence, a positive interventional outcome and a reduced rate of complications. Despite an underlying structurally normal heart, Darrieux et al. ${ }^{26}$ affirmed that PVCs arising from the ROVT (PVC-RVOT) were most likely to generate symptoms ${ }^{23} 27$. Farzaneh et al. ${ }^{28}$ noted that $80 \%$ of individuals with PVC-RVOT were free from structural cardiac pathology. Earlier research findings are in keeping with the current study. Despite the fact that patients with a low PVC burden were selected for this study, the majority had PVCs originating from the RVOT. All the subjects reported symptoms; only a single individual was noted to have underlying cardiac structural abnormality. These observations are disparate to those found by Xu et al. ${ }^{29}$; these authors reported the presence of a high PVC burden in patients with PVC-RVOT.

It has been demonstrated that individuals with an impaired LVEF have a greater average PVC burden than those with an LVEF within the normal range, i.e. $29-37 \%$ and $8-13 \%$, respectively ${ }^{11}{ }^{14}$. Cardiomyopathy precipitated by PVCs has been occasionally noted in subjects with relatively few PVCs ${ }^{8}$. The data presented in the current study are in agreement, in that the participants with a mean PVC burden of $8 \%$ demonstrated a normal LEVF of $62.9 \%$ and LVEDd of $43.8 \mathrm{~mm}$ on echocardiography. These two parameters remained stable following RFA indicative of the absence of PVC-related cardiac impairment prior to the procedure.

Only a single individual was noted to have a low LVEF of $29 \%$ and dilated LVEDd of $67 \mathrm{~mm}$ prior to RFA owing to concurrent coronary artery disease. Subjects in whom a reduced LVEF was identified only demonstrated a rise of LVEF of up to $15 \%$ on the yearly follow-up scan, despite a positive outcome from RFA defined by the drop in PVC burden from $9.8 \%$ to $0 \%$. PVC-related cardiomyopathy is presumed if the diminished baseline LVEF rises by a minimum of $15 \%$ or returns to the normal range, i.e. $>50 \%$, following 
RFA $^{11}$. It was therefore surmised that the cardiac impairment seen in this individual was unlikely to be a result of PVCs or concomitant structural cardiac pathology.

Changes in symptom burden and quality of life in low burden PVC patients 12 months after ablation

It was observed that both symptom load and life quality improved within the year after RFA in patients with a low initial PVC burden, which was in keeping with the recorded reduction in PVC burden following the procedure. Earlier studies have additionally demonstrated the success of RFA in diminishing PVC frequency, and in alleviating symptoms and enhancing life quality in patients with a relatively high PVC burden ${ }^{130}$. In one study in which 30 patients with a high PVC burden causing symptoms underwent RFA, PVC frequency and symptoms were reduced and a superior life quality was reported a year following the intervention ${ }^{24}$; these were similar results to those obtained in the current study on subjects with a low PVC burden. At follow-up, two-thirds of patients were symptom-free; in the remaining third, their symptoms had been ameliorated and they reported no physical or psychological disruptions to their life quality.

Changes in PVC burden 12 months after ablation

Earlier work has presented evidence to demonstrate that RFA has a higher efficacy than pharmaceutical therapy for the eradication of PVCs. However, these studies were generally performed on individuals with a high PVC burden. Specialist recommendations published by the European Heart Rhythm Association (2014) relating to ventricular dysrhythmias have advised RFA in individuals who remain symptomatic in the face of medical therapy or who have non-permanent impaired left ventricular function related to PVCs.

The current study has demonstrated a fall in PVC prevalence a year following RFA as documented using 24-hour Holter monitoring. These data are in keeping with former research on populations with a high PVC burden $8,12,13,31$. In a study of 30 patients, a fall in the high PVC burden of $23.1 \%$ to $1 \%$ was noted at 3-4 months follow-up ${ }^{32}$. A similar decrease in high PVC burden, from $19 \%$ to $1.1 \%$ was noted in 50 individuals in a study published by Baser et al. ${ }^{25}$.

Recurrence incidence 12 months after ablation of low burden PVC patients

Statistics have revealed that individuals who exhibit over 12 PVCs on a daily basis have an increased mortality rate from all etiologies and a higher number of in-patient admissions for cardiac pathologies, including myocardial infarctions, when judged against subjects with a lower number of PVCs ${ }^{6}$. A RFA intervention is deemed successful if the PVC burden were diminished by $80 \%$ of the baseline total ${ }^{1125}$. There is a range of parameters that have been used to determine PVC resumption following RFA; current research has predominantly used populations with a high PVC burden. The definition of recurrence in this study was failure to decrease the PVC burden by $80 \%$ of the baseline value as recognized by 24 -hour Holter electrocardiography, or the resumption of PVC-related symptomatology documented during the Holter monitoring period. 
According to the above criteria, $26.6 \%$ were noted to have recurrent PVCs a year after RFA. A number of earlier researchers have demonstrated the frequency of PVC recurrence following RFA to be between 10$20 \%$, although it was slightly higher in one study ${ }^{24}$, which documented a recurrence rate of $26 \%(8 / 30$ participants). Patients with recurring PVCs reported symptoms, although on additional analysis, the residual symptomatology was less intense than prior to RFA and failed to impact life quality. Those who were deemed to have recurrent PVCs owing to less than $80 \%$ PVC reduction but who were symptom-free implied that the subjective parameters relating to the presence or absence of symptoms could not be utilized in isolation.

Strengths and limitations of the study

The life quality of individuals with a low PVC burden who underwent PVC was assessed. This research therefore forms the foundation for establishing the application of RFA in this patient cohort. Subjective bias from patients self-completing the questionnaire cannot be entirely excluded. Furthermore, the Holter system utilized was only a seven-lead system, which has restrictions with respect to recognizing the focal loci of PVCs in those individuals in whom recurrence was suspected. This is significant, as RFA may have been effective in ablating earlier PVCs but detected de novo PVCs may have arisen from a different site.

\section{Conclusions}

In patients with a low PVC burden, RFA offered a symptom burden reduction and enhanced life quality after a year following the intervention. The PVC burden was diminished at this juncture; PVC recurrence was identified in $26.6 \%$ subjects.

Thus, it can be concluded that patients with a low PVC burden but who experience symptoms should be treated with RFA. A more prolonged monitoring period is favored, i.e. a minimum of 48 hours. Additional studies are merited to establish the impact of RFA on the burden of symptoms and life quality in patients with only a low PVC burden; patients should be followed up long-term and studies should incorporate larger population sizes.

\section{Declarations}

Ethics approval and consent to participate

The Commission on Health Research Ethics Medical Faculty of Hasanuddin University RSPTN gave ethical approval for this research (protocol number: UH20020106). All study subjects were provided information pertaining to the aims and objectives of the study; their anonymity was guaranteed. Informed consent was obtained from all participants at baseline. Subjects were reassured that they could leave the study at any juncture.

Consent for Publication 
Not applicable

Availability of data and material

Datasets generated during and/or analysed during the current study are available from the corresponding author on reasonable request.

Competing interests

The author (s) declare no competing interests.

Funding

No external monetary support was required for this study.

Authors contributions

The manuscript was written by all the authors. MA, PK and MNIS were responsible for study design, data collection, analysis and interpretation, and manuscript drafting. PT, IJP and SMZ contributed to data analysis and interpretation, and the writing of the manuscript. The final manuscript was read, critiqued, revised and ultimately approved by all authors.

Acknowledgements

The authors would like to express their sincere gratitude towards the Integrated Heart Center Wahidin Sudirohusodo Hospital, Makassar, Indonesia for their cooperation in this study.

\section{References}

1. Huang CX, Liang JJ, Yang B, et al. Quality of life and cost for patients with premature ventricular contractions by radiofrequency catheter ablation. PACE - Pacing Clin Electrophysiol. 2006;29(4):343-350. doi:10.1111/j.1540-8159.2006.00351.x

2. WHO, Committee. Rehabilitation of Patients with Cardiovascu-Lar Disease.Pdf.; 1964.

3. Amir M, Mappangara I, Setiadji R, Zam SM. Characteristics and Prevalence of Premature Ventricular Complex: A Telemedicine Study. Cardiol Res. 2019;10(5):285-292. doi:10.14740/cr887

4. Andre G. Ventricular Ectopic Beats. Published online 2006. doi:10.1136/hrt.2005.067843

5. Lee A, Denman R, Haqqani HM. Ventricular Ectopy in the Context of Left Ventricular Systolic Dysfunction: Risk Factors and Outcomes Following Catheter Ablation. Hear Lung Circ. 2019;28(3):379388. doi:10.1016/j.hlc.2018.01.012 
6. Lin CY, Chang SL, Lin YJ, et al. An observational study on the effect of premature ventricular complex burden on long-term outcome. Med (United States). 2017;96(1).

doi:10.1097/MD.0000000000005476

7. Shanmugam N, Chua TP, Ward D. "Frequent" ventricular bigeminy - A reversible cause of dilated cardiomyopathy. How frequent is "frequent"? Eur J Heart Fail. 2006;8(8):869-873.

doi:10.1016/j.ejheart.2006.02.011

8. Yarlagadda RK, Iwai S, Stein KM, et al. Reversal of cardiomyopathy in patients with repetitive monomorphic ventricular ectopy originating from the right ventricular outflow tract. Circulation. 2005;112(8):1092-1097. doi:10.1161/CIRCULATIONAHA.105.546432

9. Kanei Y, Friedman M, Ogawa N, Hanon S, Lam P, Schweitzer P. Frequent premature ventricular complexes originating from the right ventricular outflow tract are associated with left ventricular dysfunction. Ann Noninvasive Electrocardiol. 2008;13(1):81-85. doi:10.1111/j.1542-474X.2007.00204.x

10. Taieb JM, Maury P, Shah D, et al. Reversal of dilated cardiomyopathy by the elimination of frequent left or right premature ventricular contractions. J Interv Card Electrophysiol. 2007;20(1-2):9-13. doi:10.1007/s10840-007-9157-2

11. Baman TS, Lange DC, llg KJ, et al. Relationship between burden of premature ventricular complexes and left ventricular function. Hear Rhythm. 2010;7(7):865-869.

doi:10.1016/j.hrthm.2010.03.036

12. Takemoto $\mathrm{M}$, Yoshimura $\mathrm{H}, \mathrm{Ohba} \mathrm{Y}$, et al. Radiofrequency catheter ablation of premature ventricular complexes from right ventricular outflow tract improves left ventricular dilation and clinical status in patients without structural heart disease. J Am Coll Cardiol. 2005;45(8):1259-1265.

doi:10.1016/j.jacc.2004.12.073

13. Bogun F, Crawford T, Reich S, et al. Radiofrequency ablation of frequent, idiopathic premature ventricular complexes: Comparison with a control group without intervention. Hear Rhythm. 2007;4(7):863-867. doi:10.1016/j.hrthm.2007.03.003

14. Hasdemir C, Ulucan C, Yavuzgil O, et al. Tachycardia-induced cardiomyopathy in patients with idiopathic ventricular arrhythmias: The incidence, clinical and electrophysiologic characteristics, and the predictors. J Cardiovasc Electrophysiol. 2011;22(6):663-668. doi:10.1111/j.1540-8167.2010.01986.x

15. Aliot EM, Stevenson WG, Almendral-Garrote JM, et al. EHRA/HRS expert consensus on catheter ablation of ventricular arrhythmias. Europace. 2009;11(6):771-817. doi:10.1093/europace/eup098

16. Al-Khatib SM, Stevenson WG, Ackerman MJ, et al. 2017 AHA/ACC/HRS Guideline for Management of Patients With Ventricular Arrhythmias and the Prevention of Sudden Cardiac Death. Vol 138.; 2018. doi:10.1161/CIR.0000000000000549 
17. Priori SG, Blomström-Lundqvist C, Mazzanti A, et al. 2015 ESC Guidelines for the management of patients with ventricular arrhythmias and the prevention of sudden cardiac death: The Task Force for the Management of Patients with Ventricular Arrhythmias and the Prevention of Sudden Cardiac Death of the Europe. Europace. 2015;17(11):1601-1687. doi:10.1093/europace/euv319

18. Yokokawa M, Good E, Crawford T, et al. Recovery from left ventricular dysfunction after ablation of frequent premature ventricular complexes. Hear Rhythm. 2013;10(2):172-175.

doi:10.1016/j.hrthm.2012.10.011

19. Lee D, Hoffmayer KS, Hsu JC, et al. Long-term mode and timing of premature ventricular complex recurrence following successful catheter ablation. J Interv Card Electrophysiol. 2019;55(2):153-160. doi:10.1007/s10840-019-00520-3

20. Walfridsson U, Arestedt K, Stromberg A. Development and validation of a new Arrhythmia-Specific questionnaire in Tachycardia and Arrhythmia ( ASTA) with focus on symptom burden. Published online 2012:1-10.

21. Ulla W, Kristofer A. Development and Validation of an Arrhythmia-Specific Scale in Tachycardia and Arrhythmia With Focus on Health-Related Quality of Life. 2015;30(2):98-108. doi:10.1097/JCN.0000000000000149

22. Kerola T, Dewland TA, Vittinghoff E, Heckbert SR, Stein PK, Marcus GM. Modi fi able Predictors of Ventricular Ectopy in the Community. Published online 2018:1-13. doi:10.1161/JAHA.118.010078

23. Latchamsetty R, Yokokawa M, Morady F, et al. Multicenter outcomes for catheter ablation of idiopathic premature ventricular complexes. JACC Clin Electrophysiol. 2015;1(3):116-123. doi:10.1016/j.jacep.2015.04.005

24. Krittayaphong R, Sriratanasathavorn C, Bhuripanyo K, et al. One-year outcome after radiofrequency catheter ablation of symptomatic ventricular arrhythmia from right ventricular outflow tract. Am J Cardiol. 2002;89(11):1269-1274. doi:10.1016/S0002-9149(02)02324-X

25. Baser K, Bas HD, Belardi D, et al. Predictors of outcome after catheter ablation of premature ventricular complexes. J Cardiovasc Electrophysiol. 2014;25(6):597-601. doi:10.1111/jce.12400

26. Darrieux FCC, Scanavacca MI, Hachul DT, et al. Radiofrequency catheter ablation of premature ventricular contractions originating in the right ventricular outflow tract. Arq Bras Cardiol. 2007;88(3):236243. doi:10.1590/S0066-782X2007000300003

27. Wang $\mathrm{J}$ sheng, Shen $\mathrm{Y}$ gen, Yin $\mathrm{R}$ peng, et al. The safety of catheter ablation for premature ventricular contractions in patients without structural heart disease. BMC Cardiovasc Disord. 2018;18(1):1-6. doi:10.1186/s12872-018-0913-2 
28. Farzaneh-Far A, Lerman BB. Idiopathic ventricular outflow tract tachycardia. Heart. 2005;91(2):136138. doi:10.1136/hrt.2004.033795

29. Xu W, Li M, Chen M, et al. Effect of burden and origin sites of premature ventricular contractions on left ventricular function by 7-day Holter monitor. J Biomed Res. 2015;29(6):465-474. doi:10.7555/JBR.29.20150032

30. Pytkowski M, Maciag A, Jankowska A, et al. Quality of life improvement after radiofrequency catheter ablation of outfl ow tract ventricular arrhythmias in patients with structurally normal hearts. Acta Cardiol. 2012;67(2):153-159. doi:10.1080/ac.67.2.2154205

31. Sekiguchi Y, Aonuma K, Yamauchi Y, et al. Chronic hemodynamic effects after radiofrequency catheter ablation of frequent monomorphic ventricular premature beats. J Cardiovasc Electrophysiol. 2005;16(10):1057-1063. doi:10.1111/j.1540-8167.2005.40786.x

32. El Kadri M, Yokokawa M, Labounty $\mathrm{T}$, et al. Effect of ablation of frequent premature ventricular complexes on left ventricular function in patients with nonischemic cardiomyopathy. Hear Rhythm. 2015;12(4):706-713. doi:10.1016/j.hrthm.2014.12.017 